\title{
FULLY NONLINEAR FLOW OVER SUCCESSIVE OBSTACLES: HYDRAULIC FALL AND SUPERCRITICAL FLOWS
}

\author{
SHAUN R. BELWARD ${ }^{1}$
}

(Received 29 August 1996; revised 10 May 1997)

\begin{abstract}
In this paper we consider the flow of an incompressible, inviscid and homogeneous fluid over two obstacles in succession. The flow is assumed irrotational and solutions are sought in which a hydraulic fall occurs over the first obstacle with supercritical flow over the second. The method used to solve the problem is capable of calculating flows over topography of any shape.
\end{abstract}

\section{Introduction}

The knowledge about fully nonlinear flow over obstacles has increased considerably in the last decade or two. Much of this work has concentrated on finding flow solutions over a single obstacle and most often this obstacle has had a special shape. For example, Forbes [4] considered flow over a semi-elliptical obstacle, Forbes and Schwartz [7] considered flow over a semi-circle and Dias and Vanden-Broeck [3] studied flow over a triangular weir. The advantage of using these special shapes is that a conformal mapping can be used to cater for singularities that occur on the bottom surface. More recently, techniques for calculating flow over arbitrary bottom topography have been developed. These include the work of King and Bloor [8] who used a generalization of the Schwartz-Christoffel transformation, the work of Belward and Forbes [1] and [2] who used a direct boundary integral solution method and the work of Read, Belward and Higgins [10] who used a series solution technique. It is important to note that although these authors presented techniques capable of calculating flow over arbitrary topography, there has yet been no work devoted to the study of fully nonlinear flow over successive obstacles.

\footnotetext{
'Department of Mathematics and Statistics, James Cook University of North Queensland, Townsville, Queensland 4811, Australia.

(C) Australian Mathematical Society 1999, Serial-fee code 0334-2700/99
} 
In this paper we will utilize the boundary integral technique of Belward and Forbes [1] and [2] to look at flow over successive obstacles. It is well-known that when irrotational flow of an inviscid, incompressible fluid occurs over a single obstacle there are three possible flow configurations. Which flow configuration exists depends largely on the Froude number, defined as $F=u / \sqrt{g h}$, where $u$ and $h$ are respectively the speed and depth of fluid and $g$ is the acceleration due to gravity. The first flow configuration that may occur is that known as downstream wave flow, where $F<1$ throughout the fluid, with wave-free flow ahead of the obstacle and a train of almost periodic waves downstream. Meteorologists refer to such flow as "lee-wave" flows, as they occur in the atmosphere, produced by wind over mountains. The second possible flow configuration is known as critical flow, or as a hydraulic fall, where the fluid is wave-free ahead of the obstacle with $F<1$ and then cascades in a "waterfall-like" way over the obstacle so $F=1$ somewhere over the obstacle with $F>1$ in a thin layer of relatively high speed fluid downstream of the obstacle. This flow is also wellknown to meteorologists (as the severe downslope windstorm), as this configuration is not uncommon in the atmosphere at the foothills of certain mountain ranges. Belward and Forbes [2] discuss in further detail a simple model for the atmosphere which adequately predicts the flows mentioned above. The third possible configuration of flow over a single obstacle is that known as supercritical flow, where the fluid flows with $F>1$ on either side of the obstacle and the free surface of the fluid is symmetric about the centre of the obstacle, if it itself is symmetric about its centre.

Given the three basic flow configurations possible for flow over a single obstacle, we can postulate what flow configurations may occur during flow over two obstacles. If flow occurs with $F<1$ on both sides of the first obstacle we would expect that a train of almost periodic waves could be generated by the first obstacle. These waves would then interact with the second obstacle (which can be thought of as producing its own train of lee waves), with one possible outcome being that the waves could be amplified or diminished (by addition of waves produced by the second obstacle in or out of phase with those produced by the first) so that $F<1$ in the lee of the second obstacle. The linear theory discussed in Belward and Forbes [1] certainly predicts this. Another possible outcome is that if the second obstacle is the correct height for the Froude number of the flow upstream from it, critical flow could occur over the second obstacle, so that $F>1$ in the lee of the second obstacle. This has been observed experimentally in the work of Pratt [9]. If the waves produced by the first obstacle were of high amplitude, the second obstacle may cause these waves to break and thus no steady flow would exist. When $F>1$ everywhere, we would expect supercritical flow to occur over each of the obstacles, so that the flow would be almost symmetric within some neighborhood about the centre of each obstacle.

The final possible flow configuration is that where critical flow occurs over the first obstacle, so $F<1$ in the region upstream of it, with supercritical flow $(F>1)$ in the 
lee of this obstacle and over the second. Again, this has been observed experimentally by Pratt [9]. In this paper we examine such a flow configuration. Flows involving waves between the obstacles are more difficult to calculate and are worthy of discussion in a separate publication.

In Section 2 we present the mathematical formulation of the problem and briefly discuss the numerical solution procedure. In Section 3 our results will be presented and comparisons made with results for supercritical flow over a semicircular obstacle in the work by Forbes and Schwartz [7]. Finally in Section 4 we summarize our findings and discuss areas for further research.

\section{Formulation and numerical method}

We consider irrotational flow of a single layer of inviscid, incompressible fluid of constant density over two obstacles in succession. Far upstream of the first obstacle the fluid flows with uniform height, $H$, and with velocity $c i$. Variables in the problem are nondimensionalised with respect to $H$ and $c$ and we work purely in a nondimensional system. The profile of the obstacles is given by $y=B(x)$, where $x$ and $y$ are horizontal and vertical coordinates respectively. The first obstacle, in the region $x<0$, has dimensionless height $h_{1}$ and the second, in the region $x>0$, has dimensionless height $h_{2}$. The surface of the fluid is denoted by $y=S(x)$ and is unknown at the outset. Only flow stationary with respect to the obstacles is considered, so partial derivatives with respect to time are identically zero. The fluid system prior to nondimensionalisation is shown in Figure 1, for an actual solution obtained with the method presented later in this section.

The equations describing this problem are easily obtained from Belward and Forbes [2] by setting the parameter $\beta$ used in that work to unity. This has the effect of removing the upper layer, used there to model the upper atmosphere. For completeness we briefly summarize the equations.

There are two dimensionless parameters describing the flow. There are the upstream Froude number

$$
F=\frac{c}{\sqrt{g H}}
$$

and $V$ the fluid speed far downstream of the second obstacle. Note that for critical flow, $V>1$.

We introduce the velocity potential $\phi$ so that $\mathbf{q}=\nabla \phi$ is the velocity vector. Continuity then gives

$$
\nabla^{2} \phi=0
$$




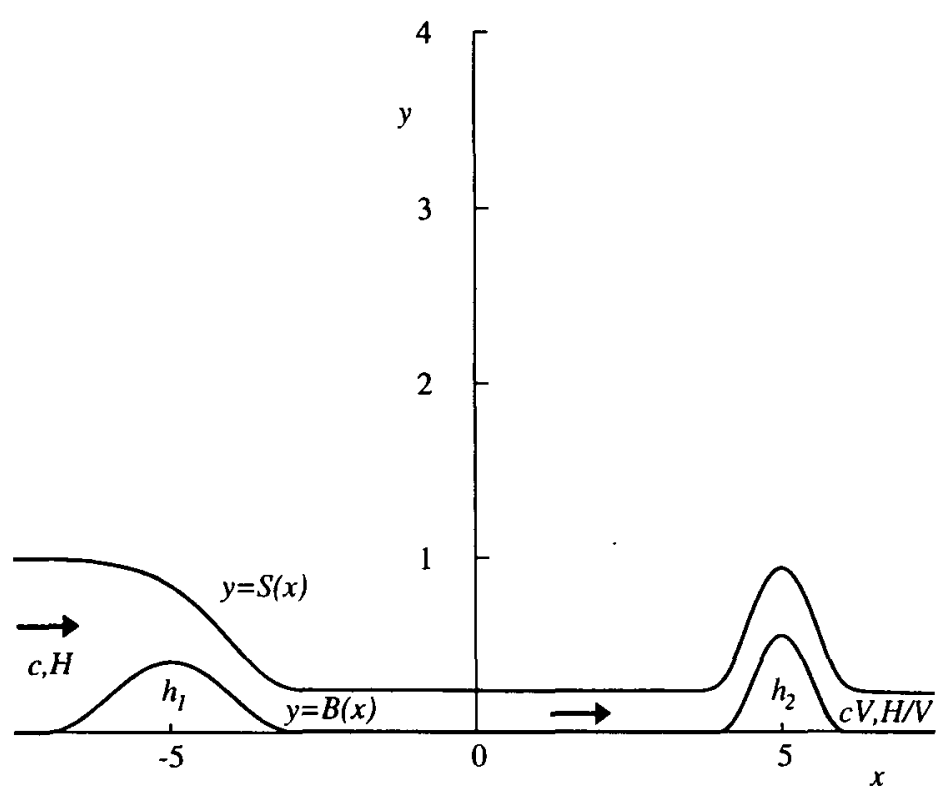

FIGURE 1. A diagram of the fluid system, flowing over two obstacles represented by $y=B(x)$, the first with height $h_{1}$ and the second with height $h_{2}$. The surface $y=S(x)$ is to be obtained; once known, all other flow quantities can be determined. This profile is a portion of an actual solution obtained with $h_{1}=0.4, h_{2}=0.55$; the upstream Froude number was calculated to be $F=0.297$.

The free surface of the fluid is a streamline, thus

$$
\nabla \phi \cdot \mathbf{n}=0 \quad \text { on } \quad y=S(x) .
$$

The surface of the obstacle is a streamline, thus

$$
\nabla \phi \cdot \mathbf{n}=0 \quad \text { on } \quad y=B(x) .
$$

Here $\mathbf{n}$ refers to the normal vector to the free surface and the bottom respectively. The upstream and downstream conditions are

$$
\begin{aligned}
& \mathbf{q} \rightarrow \mathbf{i}, \quad S(x) \rightarrow 1 \quad \text { as } \quad x \rightarrow-\infty \\
& \mathbf{q} \rightarrow V \mathbf{i}, \quad S(x) \rightarrow 1 / V \quad \text { as } \quad x \rightarrow \infty .
\end{aligned}
$$

Parameterizing the interface using arclength $s$, as in Belward and Forbes [2], gives the Bernoulli equation in the form

$$
\frac{1}{2} F^{2}\left(\frac{d \phi}{d s}\right)^{2}+y(s)=\frac{1}{2} F^{2}+1
$$


and the arclength condition

$$
\left(\frac{d x}{d s}\right)^{2}+\left(\frac{d y}{d s}\right)^{2}=1,
$$

both on the free surface. The problem therefore is to solve Equation (1) subject to the boundary conditions (2), (3), (6) and (7) and the asymptotic conditions (4) and (5).

In order to calculate the location of the unknown surface, the analytic function $\chi(z)=u(z)-i v(z)-1$ is formed, where $u(z)$ and $v(z)$ are the horizontal and vertical components of $\mathbf{q}$ at the point $z=x+i y$. This function has the property that it vanishes at upstream and downstream infinity. Two integro-differential equations are now obtained, each arising from Cauchy's integral formula

$$
\oint_{\Gamma} \frac{\chi(z) d z}{z-z^{*}}=0
$$

where the contour $\Gamma$ consists of the free surface, the bottom surface and the lines $x= \pm L$ with $L \rightarrow \infty$. The first integro-differential equation uses $z^{*}=z(s)$, a point on the free surface and has added to the contour a semi-circle of vanishingly small radius around that point. The second equation uses $z^{*}=x^{*}+i B\left(x^{*}\right)$, a point on the bottom surface and has added to the contour a semi-circle of vanishingly small radius around that point. In each case the desired equation is obtained by taking the imaginary part of Equation (8) and using the boundary conditions (2) and (3). Full details are contained in Belward and Forbes [2].

Finding the location of the free surface now requires a solution to be found to the two equations resulting from Equation (8), subject to the Bernoulli equation (6), the upstream and downstream asymptotic conditions, Equations (4) and (5) and the arclength condition (7).

The numerical solution procedure is exactly that used in Belward and Forbes [2]. We describe the procedure in brief. The free surface is sought at $N$ equally spaced (with respect to arclength) points $s_{1}, s_{2}, \ldots, s_{N}$, with $s_{1}$ and $s_{N}$ approximating $-\infty$ and $\infty$ respectively. A guess is made at $y^{\prime}\left(s_{2}\right), \ldots, y^{\prime}\left(s_{N}\right)$, with $y^{\prime}\left(s_{1}\right)=0$ used to satisfy uniform flow upstream. It is also necessary to define a grid on the bottom surface $y=B(x) . M$ points $x_{1}, x_{2}, \ldots, x_{M}$ are chosen, which are spaced equally with respect to the $x$-axis. We iterate as follows.

1. Integrate $y^{\prime}\left(s_{j}\right)$ to find $y\left(s_{j}\right)$ using $y\left(s_{1}\right) \approx y(-\infty)=1$.

2. Use the arclength condition (7) to calculate $x^{\prime}\left(s_{j}\right)$. Integrate to obtain $x\left(s_{j}\right)$ using $x\left(s_{1}\right)=s_{1}$.

3. Use the Bernoulli equation (6) to find $\phi\left(s_{j}\right), j=1, \ldots, N$.

4. Use the second integro-differential equation resulting from (8) by discretising it after removal of singularity by subtraction. Solve for $u\left(x_{j}\right), j=2, \ldots, M$ 
at the $M-1$ points $x^{*}=x_{j+1 / 2}=\left(x_{j}+x_{j+1}\right) / 2$. The trapezoidal rule is used for integration.

5. The first integro-differential equation resulting from (8) is now available as a cost function, as all flow quantities are known. This equation is used to update the current approximation to $y^{\prime}\left(s_{j}\right)$ until the norm of the cost function falls below some predefined value.

The trapezoidal rule is used for all integration because of its ease of implementation. The use of Simpson's rule of cubic splines may increase the domain of convergence slightly. However, as the results produced here are of high quality (as is shown later), this is seen as unnecessary.

\section{Results}

In this section we present the results obtained using the numerical method outlined in the previous section. Before we proceed we show some properties (noted by Forbes [5]) of the flow we are investigating. Combining the downstream conditions (5) and the Bernoulli equation (6) we can determine the upstream Froude number in terms of the downstream fluid speed, thus

$$
F=\sqrt{\frac{2}{V(V+1)}} .
$$

Also we can determine the downstream Froude number, $F_{D S}$, in terms of the upstream Froude number:

$$
F_{D S}=\frac{c V}{\sqrt{(g H) / V}}=F V^{3 / 2}=\frac{\sqrt{2} V}{\sqrt{V+1}} .
$$

The algorithm described in Section 2 was implemented on a computer and used to study fully nonlinear flow over two obstacles. The topography used had the profile

$$
B(x)= \begin{cases}\frac{h_{1}}{2}\left(1+\cos \left[\frac{\pi(x+5)}{L_{1}}\right]\right), & -L_{1}-5 \leq x \leq L_{1}-5, \\ \frac{h_{2}}{2}\left(1+\cos \left[\frac{\pi(x-5)}{L_{2}}\right]\right), & -L_{2}+5 \leq x \leq L_{2}+5, \\ 0, \quad \text { otherwise } & \end{cases}
$$

where $h_{1}$ and $h_{2}$ represent the heights of each obstacle, while $L_{1}$ and $L_{2}$ refer to the half-lengths of each obstacle. Thus the first obstacle was centered on $x=-5$ and the second on $x=5$. 
Solutions were obtained for a wide range of obstacle heights and widths. The procedure was as follows. The height of the first obstacle was fixed while the height of the second obstacle was increased from zero until the algorithm was unable to compute any further solutions. The initial guess used when the second obstacle was absent (that is when $h_{2}=0$ ) was a solution obtained using the method described by Belward and Forbes [2] with the parameter $\beta$ chosen as unity. The initial guess for each nonzero $h_{2}$ was the solution obtained for the previous $h_{2}$. In all cases presented here solutions have been obtained using $N=M$ and have been deemed to be found when the norm of the cost function falls below $10^{-9}$. Convergence testing by observing the free surface profiles as $N$ and $M$ increase shows that the solutions tend to a limiting surface profile for sufficiently large $N$ and $M$.

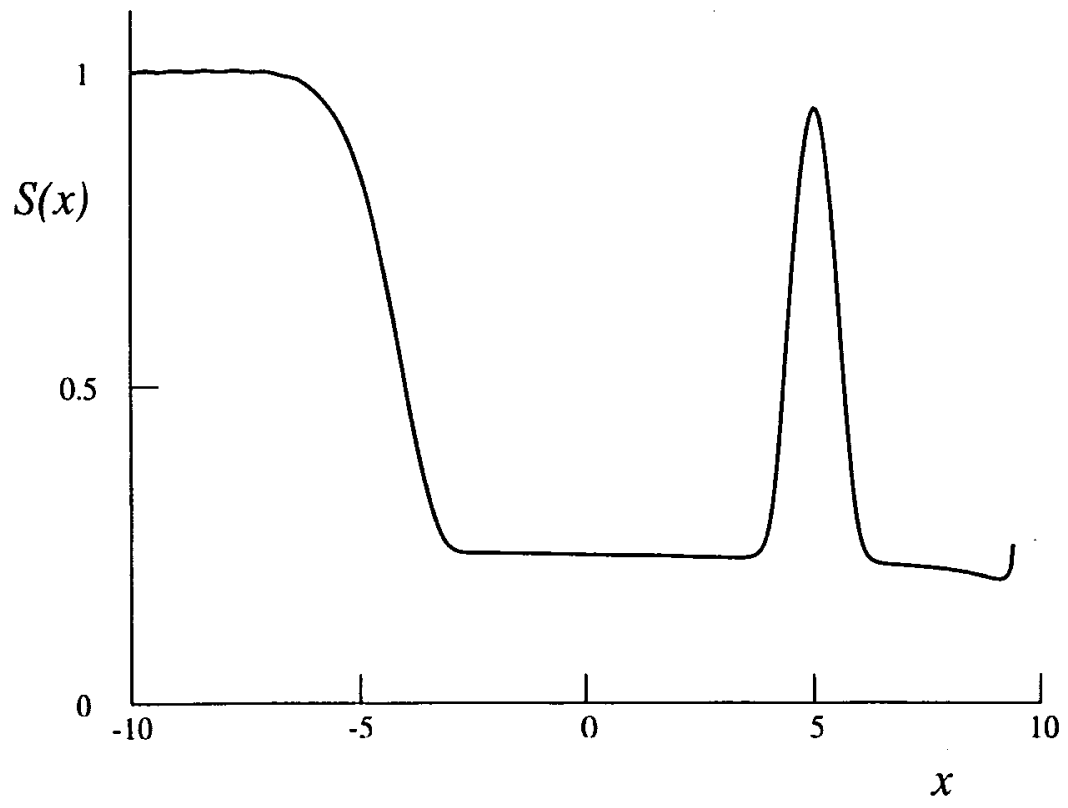

FIGURE 2. The computed free-surface profile for $h_{1}=0.4, h_{2}=0.55, L_{1}=2$ and $L_{2}=1$. 321 points were used to discretise the free surface.

Figure 2 shows a typical solution, obtained using 321 points on the free surface. In this case the first obstacle had height $h_{1}=0.4$ and half-width $L_{1}=2$, with the second having height $h_{2}=0.55$ and half-width $L_{2}=1$. The upstream Froude number was calculated to be $F \approx 0.297$ with the solution clearly showing a hydraulic fall over the first obstacle while near symmetric supercritical flow is observed over the second. Upstream of the first obstacle the solution shows waves of very small amplitude. These are common in work such as this and are a consequence of forcing uniform flow at the left side of the computational domain, when in reality it occurs at upstream 
infinity. Downstream of the second obstacle we note an inconsistency on the free surface which is a further consequence of imposing a finite computational domain.

It was found that the downstream Froude number $F_{D S}$ depended only on the height of the first obstacle. Also as $h_{1}$ increased, $F_{D S}$ increased and $F$ decreased, as results for flow over a single obstacle would predict. For a given $h_{1}$, theory suggests that the maximum possible $h_{2}$ is determined by the height at which the flow above the second obstacle would be such that a crest with an angle of $120^{\circ}$ had formed on the surface of the fluid. At this crest a stagnation point would occur so that the maximum surface elevation (the height of the crest) found from Equation (6) would be

$$
S_{\text {stag }}=\frac{1}{2} F^{2}+1
$$

This phenomenon is discussed fully in Forbes and Schwartz [7].

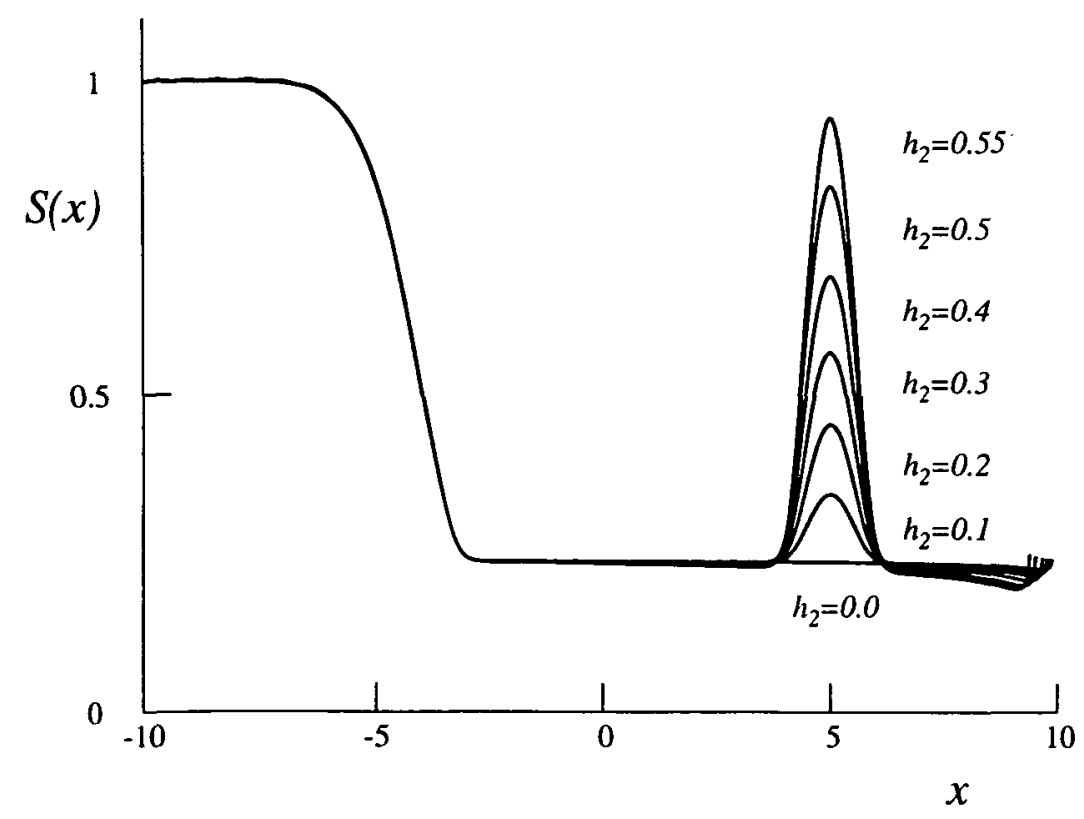

FIGURE 3. Computed free-surface profiles for $h_{1}=0.4$ and various $h_{2}$ as indicated.

Figure 3 shows the effect on the free surface of increasing $h_{2}$ while $h_{1}$ is fixed. Each of the curves in Figure 3 represents the free surface at a different height of the second obstacle, as indicated to the right of each crest. The first obstacle had height $h_{1}=0.4$ while $L_{1}=2$ and $L_{2}=1$ were fixed half-lengths. Each free surface was computed using 321 points. Clearly as the second obstacles increases in size, the curvature at the crest in the free surface also increases while the upstream Froude number remains almost fixed at $F \approx 0.297$. Equation (11) shows that with this Froude 
number, the stagnation height is $S_{\text {stag }} \approx 1.04$. From Figure 3 with $h_{2}=0.55$ we have a maximum surface elevation of $S_{\max } \approx 0.94$ which is about $90 \%$ of the maximum theoretical height, $S_{\text {stag }}$. To obtain flows closer to the maximum theoretical height, a technique capable of calculating surface profiles of high curvature is needed. This would probably involve clustering points near the region of high curvature and we have not proceeded to look further for these solutions.

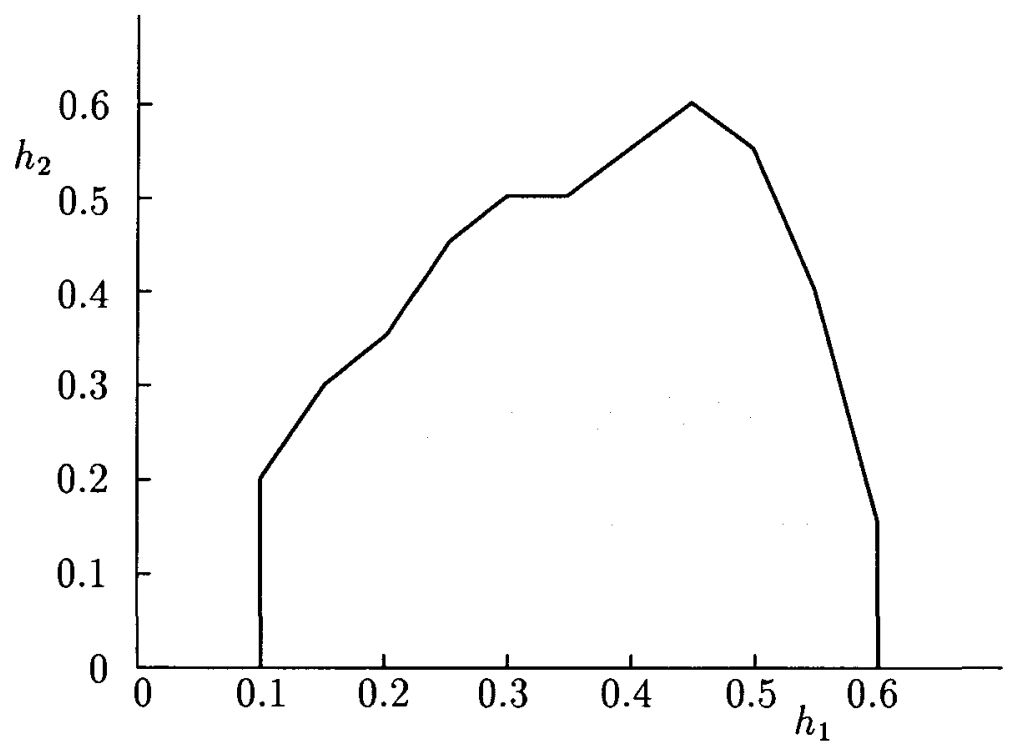

FIGURE 4. The shaded region shows the values of $h_{2}$ for which solutions could be computed, for each value of $h_{1}$.

Figure 4 shows for which heights of the second obstacle solutions could be found, given the height of the first obstacle, using 161 points on the free surface with $L_{1}$ and $L_{2}$ as in the previous figure. Note that solutions were not sought in the regions $h_{1}<0.1$ or $h_{1}>0.6$. However, there is no reason to suggest solutions would not exist in those regions if $h_{2}$ were sufficiently small. For $h_{1}<0.45$ the general trend is that as $h_{1}$ increases, so the maximum value for $h_{2}$ for which solutions exist also increases. This result is not unexpected for the following reason. The downstream Froude number, $F_{D S}$, increases as $h_{1}$ increases and $F_{D S}>1$ over the second obstacle. Forbes and Schwartz [7] showed that during supercritical flow over a semi-circular obstruction, as the Froude number of the flow increased, the maximum height of the semi-circle for which solutions could be found increased. For $h_{1}>0.45$ the maximum value for $h_{2}$ decreases rapidly. This behavior is almost certainly due to the 
high fluid speeds over the second obstacle and large free surface slope over the first obstacle. A higher-order integration scheme may obtain results for larger $h_{2}$ in this region, although it may be the case that no steady solutions exist for such high fluid speeds. Indeed in Forbes [5] the possibility of the formation of a hydraulic jump is discussed.

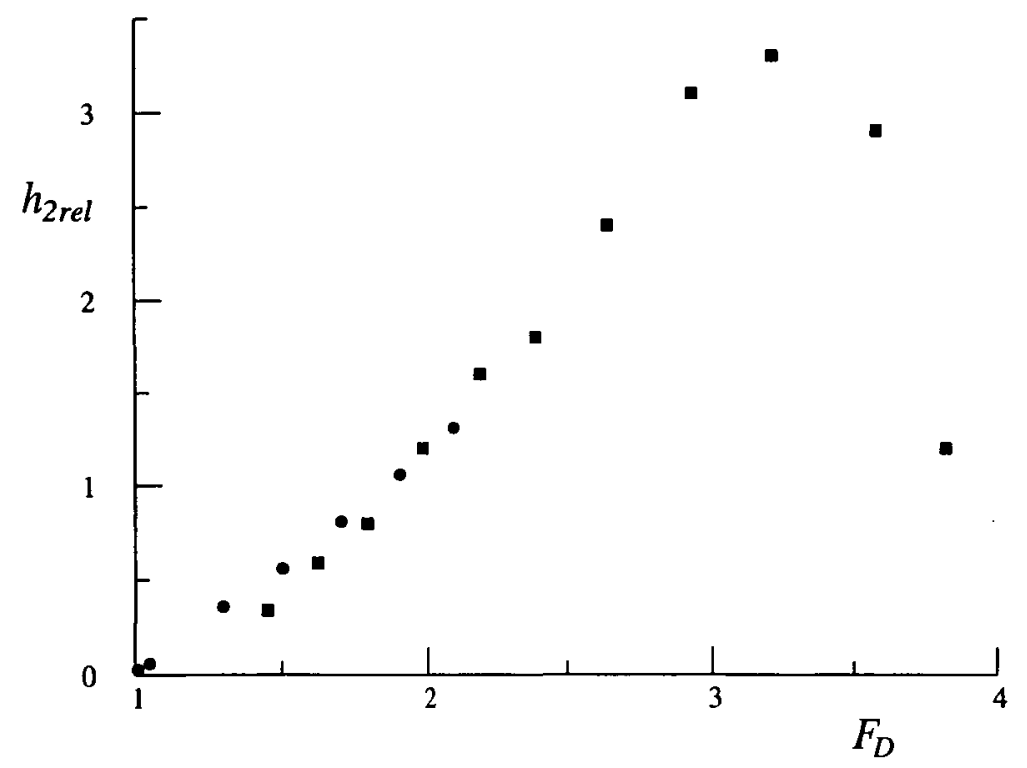

FIGURE 5. A comparison between the results obtained in this study (the squares corresponding to the downstream Froude number at which flow occurs over the second obstacle of maximum height for a particular first obstacle) and those obtained for supercritical flow over a semi-circular obstruction (the circles corresponding to the maximum height for which a solution could be computed for a given Froude number) in the study of Forbes and Schwartz [7].

Figure 5 allows a direct comparison between the results obtained in this study and that conducted by Forbes and Schwartz [7] and hence serves to validate our work. Each square of the graph represents a solution corresponding to a maximum value for $h_{2}$ for a given $h_{1}$. Thus each square corresponds to a point on the upper boundary of the shaded region in Figure 4. In obtaining Figure 5, only flow over the second obstacle is considered. The scale in the vertical direction is altered so that the fluid at the point $x=0$ has height 1 . The vertical axis on Figure 5 then gives the height of the second obstacle relative to the height of the fluid at $x=0$. Thus we define

$$
h_{2 \text { rel }}=\frac{h_{2}}{S(0)} \approx V h_{2} \text {. }
$$

The position of each square in Figure 4 is determined by the downstream Froude number, $F_{D S}$ (obtained using Equation (9)), at which the flow occurred and the 
relative height of the second obstacle. The circles in Figure 5 correspond to the results in Figure 8 of the article by Forbes and Schwartz [7] in which supercritical flow over a semi-circular obstruction was considered. Each circle represents the maximum height for a semi-circle for which the Newton process used by the authors would converge at the given Froude number. Clearly the results obtained in our study match and extend those for flow over a semi-circle. The last two squares furthest to the right in Figure 5 again show that at higher downstream fluid velocities the solution technique is not capable of calculating flows for a large $h_{2}$ as may be expected.

\section{Summary and discussion}

This paper has applied the method capable of calculating flow over arbitrary topography, developed by Belward and Forbes [1] and [2], to the problem of flow over two successive obstacles. Only a single layer of inviscid, incompressible and constant density fluid was considered and the flow was assumed irrotational. Solutions exhibiting critical flow over the first obstacle and supercritical flow over the second obstacle were the only solutions sought, although there is no doubt other solution types do exist as has been verified experimentally by Pratt [9].

The topography used in our computational experiments had the profile given by Equation (10), although other topography could easily be investigated. The Froude number at which each flow occurred was determined almost entirely by the height of the first obstacle; as the height of this obstacle increased, so the downstream Froude number increased. In general, at higher downstream Froude numbers we are able to obtain solutions for higher second obstacles. Ultimately the height of the second obstacle is limited by the formation in the flow above the obstacle of a crest of $120^{\circ}$. If the first obstacle was too high, fluid speeds downstream became large and the numerical procedure was unable to compute solutions for second obstacles as high as would otherwise be expected. Comparison with the results of Forbes and Schwartz [7] shows that, in the neighborhood of the second obstacle, the supercritical flow over that obstacle behaves in exactly the way supercritical flow over a semi-circular obstacle behaves. This comparison served to validate our results.

As noted above, configurations in which critical flow occurs over the first obstacle and supercritical flow over the second are not the only type to be observed. Work is currently underway to investigate the occurrence of waves between the obstacles and in the lee of the second. Of particular interest is the possibility that waves may occur between the obstacles with wave-free flow in the lee of the lee of the second, that is, that waves produced by the first are effectively cancelled by the second. Such a flow configuration has already been computed for flow over a single semi-ellipse by Forbes [4]. 
The importance of knowledge of flow over successive obstacles is unquestioned in the meteorological community and work is also underway to utilize the results obtained here into a model for sheared flow over topography, as may occur in the atmosphere. This model has already been used to investigate the Morning Glory phenomenon which occurs in the Gulf of Carpentaria, see Forbes and Belward [6].

\section{Acknowledgments}

I acknowledge with much appreciation a Special Studies Programme from James Cook University which enabled the completion of this work. The two anonymous referees provided helpful comments and I also thank Larry Forbes of The University of Queensland for the use of his laser2Dplot plotting package.

\section{References}

[1] S. R. Belward and L. K. Forbes, "Fully nonlinear two-layer flow over arbitrary topography", $J$. Eng. Math. 27 (1993) 419-432.

[2] S. R. Belward and L. K. Forbes, "Interfacial waves and hydraulic falls: some applications to atmospheric flows in the lee of mountains", J. Eng. Math. 29 (1995) 161-179.

[3] F. Dias and J.-M. Vanden-Broeck, "Open channel flow with submerged obstructions", J. Fluid Mech. 206 (1989) 155-170.

[4] L. K. Forbes, "Non-linear, drag-free flow over a submerged semi-elliptical body", J. Eng. Math. $16(1982) 171-180$.

[5] L. K. Forbes, "Critical free-surface flow over a semi-circular obstruction", J. Eng. Math. 22 (1988) 3-13.

[6] L. K. Forbes and S. R. Belward, "Atmospheric solitary waves: some applications to the Morning Glory of the Gulf of Carpenteria", J. Fluid Mech. 321 (1996) 137-155.

[7] L. K. Forbes and L. W. Schwartz, "Free-surface flow over a semi-circular obstruction", J. Fluid Mech. 114 (1982) 299-314.

[8] A. C. King and M. I. G. Bloor, "Free surface flow of a stream obstructed by an arbitrary bed topography", Quart. J. Mech. Appl. Math. 43 (1990) 87-106.

[9] L. J. Pratt, "On nonlinear flow with multiple obstructions", J. Atmos. Sci. 41 (1984) 1214-1225.

[10] W. W. Read, S. R. Belward and P. J. Higgins, "Iterative schemes for series solutions to Laplacian free boundary problems", in Computational techniques and applications: CTAC95 (eds. R. L. May and A. K. Easton), (World Scientific, Singapore, 1996) 669-676. 